\title{
Direct Numerical Simulation of Transition due to Traveling Crossflow Vortices
}

\author{
Fei Li*, Meelan M. Choudhari ${ }^{+}$ \\ NASA Langley Research Center, Hampton, VA 23681 \\ Lian Duan ${ }^{\&}$ \\ Missouri University of Science and Technology, Rolla, MO 65409
}

Previous simulations of laminar breakdown mechanisms associated with stationary crossflow instability over a realistic swept-wing configuration are extended to investigate the alternate scenario of transition due to secondary instability of traveling crossflow modes. Earlier analyses based on secondary instability theory and parabolized stability equations have shown that this alternate scenario is viable when the initial amplitude of the most amplified mode of the traveling crossflow instability is greater than approximately 0.03 times the initial amplitude of the most amplified stationary mode. The linear growth predictions based on the secondary instability theory and parabolized stability equations agree well with the direct numerical simulation. Nonlinear effects are initially stabilizing but subsequently lead to a rapid growth followed by the onset of transition when the amplitude of the secondary disturbance exceeds a threshold value. Similar to the breakdown of stationary vortices, the transition zone is rather short and the boundary layer becomes completely turbulent across a distance of less than 15 times the boundary layer thickness at the completion of transition.

\section{Nomenclature}

$A=$ amplitude of crossflow instability mode or secondary instability mode at a given chordwise location defined as the ratio of chordwise perturbation velocity to freestream velocity

$A_{\text {init }} \quad=$ initial amplitude (measured at lower branch neutral location for primary disturbance and at $x / c=0.3$ for secondary disturbance); the amplitude is measured in terms of the peak fluctuation in chordwise velocity component at the relevant station.

AoA $=$ angle of attack, degrees

$c, C=$ wing chord length measured in the direction perpendicular to the leading edge, meters

$c_{s} \quad=$ wing chord length measured in the freestream direction, meters

$f=$ frequency of instability oscillations in Hertz

$k \quad=\quad$ ratio of secondary mode frequency in the traveling frame to the frequency of the primary, i.e., traveling crossflow mode

$M \quad=\quad$ freestream Mach number

$m \quad=$ Fourier index of frequency mode

$N \quad=$ logarithmic amplitude ratio, i.e., N-factor of linear crossflow instability or secondary instability

$n=$ Fourier index of spanwise mode

$\operatorname{Re}_{c} \quad=$ Reynolds number based on streamwise chord length

$T_{\infty} \quad=$ Freestream temperature, $\mathrm{K}$

$t \quad=$ time, seconds

$U_{\infty} \quad=$ freestream velocity, $\mathrm{m} / \mathrm{s}$

$u=$ chordwise perturbation velocity, $\mathrm{m} / \mathrm{s}$

$x, X=$ chordwise coordinate in the direction perpendicular to leading edge

$y, Y=$ wall-normal coordinate

$z, Z=$ spanwise coordinate in the direction parallel to leading edge

* Aerospace Technologist, Computational AeroScience Branch, fei.li@nasa.gov

+Aerospace Technologist, Computational AeroScience Branch, meelan.m.choudhari@nasa.gov, Associate Fellow, AIAA.

\& Assistant Professor, Mechanical and Aerospace Engineering, duanl@mst.edu, Senior Member, AIAA. 


$\begin{array}{ll}\Delta x^{+} & =\text {grid resolution in streamwise direction, in wall unit } \\ \Delta y^{+} & =\text {grid resolution in spanwise direction, in wall unit } \\ \Delta z^{+} & =\text {grid resolution in wall-normal direction, in wall unit } \\ \beta & =\text { spanwise wave number } \\ \lambda & =\text { spanwise wavelength } \\ \omega_{p} & =\text { frequency of primary traveling crossflow vortex in fixed frame of reference } \\ \omega_{s} & =\text { frequency of secondary instability in moving frame of reference } \\ \varepsilon & =\text { detune parameter for secondary instability } \\ \xi & =\text { vortex aligned coordinate in non-orthogonal system } \\ \eta & =\text { wall-normal coordinate in non-orthogonal system } \\ \zeta & =\text { spanwise coordinate in non-orthogonal system } \\ \varphi & =\text { flow field perturbation } \\ \bar{\varphi} & =\text { flow field for base flow } \\ \hat{\bar{\varphi}} & =\text { shape function of spanwise Fourier mode for stationary base flow } \\ \hat{\varphi}_{n} & =\text { shape function of spanwise Fourier mode for secondary instability } \\ \psi & =\text { flow field perturbation quantity } \\ L S I T & =\text { linear secondary instability theory } \\ P S E & =\text { parabolized stability equations } \\ N P S E & =\text { nonlinear parabolized stability equations } \\ D N S & =\text { direct numerical simulations } \\ T S & =\end{array}$

\section{Introduction}

The economic and environmental benefits of laminar flow technology via reduced fuel burn of subsonic and supersonic aircraft cannot be realized without minimizing the uncertainty in drag prediction in general and transition prediction in particular. Transition research under NASA's Aeronautical Sciences Project seeks to develop a validated set of variable fidelity prediction tools with known strengths and limitations, so as to enable sufficiently accurate transition prediction and practical transition control for future vehicle concepts. An important ingredient of this development corresponds to an understanding of the linear amplification characteristics of relevant primary instabilities, their nonlinear development, and subsequent mechanisms responsible for the onset of transition. Whereas tools based on linear stability theory, linear and nonlinear parabolized stability equations, and secondary instability analysis can predict the earlier stages of transition, numerical simulations play an important role in providing the required insights into the laminar breakdown phase that extends from the onset of transition to a fully developed turbulent boundary layer. Numerical simulations of swept wing transition due to high frequency secondary instabilities of stationary crossflow vortices were presented in earlier papers by Duan et al. ${ }^{1}$ and Choudhari et al. ${ }^{2}$ The purpose of the present paper is to extend those simulations to address the transition mechanisms involving the breakdown of traveling crossflow vortices.

In general, a swept wing boundary layer can be susceptible to various types of primary instabilities such as attachment line instability, ${ }^{3}$ stationary and traveling crossflow modes, and Tollmien-Schlichting (TS) waves. Regardless of which mode dominates the primary amplification stage, the other modes could play a role during the nonlinear stage and hence, influence the onset of transition. In aerodynamic configurations of practical interest, the breakdown of stationary crossflow vortices appears to be the more likely route to transition; ${ }^{4-8}$ however, other scenarios involving a more significant role for the traveling crossflow instability ${ }^{2,9-11}$ may become relevant when either the initial amplitudes of traveling modes become sufficiently large as a result of higher amplitude freestream disturbances or when the surface has been carefully polished to minimize the initial amplitudes of stationary crossflow modes. Wassermann and Klocker ${ }^{10}$ studied the nonlinear saturation of traveling crossflow vortices and the associated developments of secondary instability for a flat plate configuration subject to a favorable pressure gradient. They showed that the finite amplitude traveling crossflow 
vortices form stronger internal shear layers that are much closer to the wall than their stationary counterparts and that the secondary instability ${ }^{11}$ is dominated by the Z-mode type according to the terminology first introduced by Malik et al. ${ }^{4}$ In a recent paper, Choudhari et al. ${ }^{2}$ used nonlinear parabolized stability equations (NPSE) and linear secondary instability theory (LSIT) to analyze the traveling crossflow vortices and their secondary instability on a swept airfoil configuration with relevance to subsonic transports and found a similar behavior for the finite amplitude traveling crossflow modes as in Wassermann and Klocker. ${ }^{10}$ More importantly, the findings in Ref. 2 also established the existence of the $\mathrm{Y}$ mode ${ }^{4}$ of secondary instability, which becomes dominant when the amplitude of the primary traveling mode is sufficiently large. A more complete study of the secondary instability of traveling crossflow modes was presented by $\mathrm{Li}$ et al. ${ }^{12}$ Down and White ${ }^{13}$ studied the effect of freestream turbulence on the development of crossflow disturbances in a wind tunnel experiment and showed that increasing freestream turbulent levels promote the traveling crossflow vortices. Their paper also presents a thorough review of the previous work on this topic.

In a practical setting, investigating the nonlinear breakdown scenarios associated with a mixed evolution of stationary and traveling crossflow modes is perhaps more important than studying the breakdown mechanisms related to traveling crossflow modes in isolation. However, as a prelude to the more complex scenario related to the mixed mode evolution, the breakdown of secondary instability of the traveling crossflow modes alone is studied herein to gain useful insights into the resulting transition process. Previous work ${ }^{2,11}$ has shown that the traveling crossflow modes saturate at much lower amplitudes in comparison to the stationary modes and, yet, the growth of secondary instabilities of these traveling modes can be at least as strong as the secondary modes of the stationary crossflow modes with larger amplitudes. The work presented herein extends that effort to address the important issue of the breakdown process associated with the secondary instabilities of traveling crossflow modes and compare the transition zone characteristics with those encountered during the breakdown of stationary crossflow modes. ${ }^{1,2}$

This paper is organized as follows. Section II provides a summary of the secondary instability theory for traveling crossflow instabilities in a swept wing boundary layer. For additional details, the reader may refer to Refs. 12 and 14. The flow configuration of interest in this paper is outlined in Section III, along with the computational codes used in the analysis. Section IV provides a brief overview of the linear and nonlinear development of traveling crossflow instabilities and the linear evolution of associated secondary instabilities, followed by select simulation results concerning the nonlinear development of the secondary modes and the subsequent onset of transition. Concluding remarks are presented in Section V.

\section{Secondary Instability}

The basic state for secondary instability corresponds to the laminar boundary layer modified by the presence of a finite amplitude primary instability. When the primary instability corresponds to crossflow vortices, the base flow is slowly varying along the vortex axis but periodic in the spanwise direction with the same period as the spanwise wavelength of the primary crossflow vortex. These two directions are, in general, not orthogonal and the problem is best solved in a non-orthogonal coordinate system. The form of the secondary instability equations in a non-orthogonal coordinate system was derived in the context of stationary crossflow instability by $\mathrm{Li}$ and Choudhari. ${ }^{14}$ The secondary instability equations in a non-orthogonal coordinate system take the form

$$
\Gamma \frac{\partial \varphi}{\partial t}+A \frac{\partial \varphi}{\partial \xi}+B \frac{\partial \varphi}{\partial \eta}+A \frac{\partial \varphi}{\partial \zeta}=V_{\xi \xi} \frac{\partial^{2} \varphi}{\partial \xi^{2}}+V_{\eta \eta} \frac{\partial^{2} \varphi}{\partial \eta^{2}}+V_{\xi \xi} \frac{\partial^{2} \varphi}{\partial \xi^{2}}+V_{\xi \eta} \frac{\partial^{2} \varphi}{\partial \xi \partial \eta}+V_{\eta \xi} \frac{\partial^{2} \varphi}{\partial \eta \partial \xi}+V_{\xi \xi} \frac{\partial^{2} \varphi}{\partial \xi \partial \zeta},
$$

where $\varphi(\xi, \eta, \zeta)$ is a vector representing perturbation quantities of secondary instability, $\xi$ is the coordinate along the crossflow vortex axis, $\eta$ is normal to the wall and $\xi$ is the spanwise coordinate. The coefficient matrices in front of the derivatives of $\varphi$ are functions of the coordinates $(\xi, \eta, \xi)$, slow-varying in $\xi$ and periodic in $\zeta$. The perturbation variables associated with the secondary instability can then be expressed in the form

$$
\varphi(\xi, \eta, \zeta)=\phi(\eta, \zeta ; \xi) \exp \left(-i \omega_{s} t+i \alpha \xi\right)
$$


where $\phi(\eta, \xi ; \xi)$ is a 2D complex function corresponding to the mode shape of secondary instability at station $\xi$ along the axis of the primary crossflow vortex (i.e. the $\xi$ dependence of $\varphi$ is treated in a parametric sense), $\omega_{s}$ is the angular frequency of the secondary instability (treated as a real parameter in predicting the spatial evolution of secondary instability) and $\alpha$ is the complex wavenumber in the direction of the primary crossflow vortex. After substituting the modal ansatz (2) into the governing equations (1), the resulting set of equations is given by

$$
-i \omega_{s} \Gamma \phi+\left(i \alpha A+\alpha^{2} V_{\xi \xi}\right) \phi+\left(B-i \alpha V_{\xi \eta}\right) \frac{\partial \phi}{\partial \eta}+\left(C-i \alpha V_{\xi \xi}\right) \frac{\partial \phi}{\partial \zeta}=V_{\eta \eta} \frac{\partial^{2} \phi}{\partial \eta^{2}}+V_{\xi \xi} \frac{\partial^{2} \phi}{\partial \xi^{2}}+V_{\eta \xi} \frac{\partial^{2} \phi}{\partial \eta \partial \zeta}
$$

Together with appropriate boundary conditions in $\xi$ and $\xi$ directions, the equation set (3) corresponds to a $2 \mathrm{D}$, i.e., planar, partial differential equation based eigenvalue problem. Eq. 3 has coefficients that are 1-periodic in $\xi$ (where the notation $n$-periodic denotes periodic functions with a fundamental wavelength of $n$ times the corresponding wavelength of the primary crossflow mode), but its solution is not necessarily periodic in $\zeta$. It takes the following form given by the well-known Floquet theory ${ }^{11}$

$$
\phi(\eta, \zeta)=\Phi(\eta, \zeta) \exp \left(\frac{i \varepsilon \beta \zeta}{2}\right)
$$

where $\Phi$ is 1-periodic in $\zeta$ and, in general, $\varepsilon$ is a complex number that will be referred to herein as the detuning parameter. If the solutions to Eq. 3 are restricted to periodic functions of $\xi$, then $\varepsilon$ becomes a real parameter. In particular, $\phi$ is 1-periodic when $\varepsilon=0$ and 2-periodic when $\varepsilon=1$, which correspond to the fundamental and subharmonic modes, respectively, of the secondary instability. In this paper, however, we are primarily interested in the fundamental modes. Computations of subharmonic modes at selected frequencies show that such modes do not behave very differently from their fundamental counterparts other than a 180-degree phase shift between the locations of peak oscillations across a pair of adjacent crossflow vortex modes. We note that, once a solution is obtained in the non-orthogonal coordinate $\operatorname{system}(\xi, \eta, \zeta)$, it can be easily remapped to the more intuitive orthogonal system $(x, y, z)$.

For traveling crossflow vortices, the base flow (i.e., boundary layer flow plus the finite amplitude traveling crossflow perturbations) itself is unsteady. To circumvent the temporal variations in the base flow, the problem is analyzed in an inertial frame of reference traveling with the spanwise phase velocity of the crossflow vortex, which makes the base flow in the traveling frame independent of time as described by Malik et al. ${ }^{15}$ The transformation from the stationary to traveling coordinate system is given by

$$
Z=z-\omega_{p} t / \beta
$$

The stationary base flow in the traveling frame of reference can be expressed in the form

$$
\bar{\varphi}(x, y, Z)=\sum_{n=-\infty}^{\infty} \hat{\bar{\varphi}}_{n}(x, y) \exp (\operatorname{in} \beta Z),
$$

Secondary instability analysis in this traveling coordinate system is very similar to the secondary instability of a stationary crossflow mode, except that the base flow spanwise velocity is now non-zero at the wall. In the spanwise traveling frame, each linear secondary instability mode can be associated with a single fixed angular frequency, $\omega_{s}$. Just as for the secondary instability of stationary crossflow modes, ${ }^{14}$ the secondary instability of traveling crossflow modes can be expressed in terms of the Fourier series expansion 


$$
\varphi(x, y, Z, t)=\sum_{n=-\infty}^{\infty} \hat{\varphi}_{n}(x, y) \exp \left(i n \beta Z-i \omega_{s} t\right)
$$

In experiments, the measurement devices are usually fixed in the stationary frame of the wind tunnel. Naturally, we would like to inquire what frequency or frequencies would be registered by the measurement sensors? This question can be answered by substituting Eq. (5) into Eq. (7) to obtain the expression for the secondary perturbation in the fixed frame of reference:

$$
\varphi(x, y, z, t)=\sum_{n=-\infty}^{\infty} \hat{\varphi}_{n}(x, y) \exp \left(i n \beta z-\left(i n \omega_{p}+i \omega_{s}\right) t\right)
$$

whereas the base flow in this fixed frame of reference is given by

$$
\bar{\varphi}(x, y, z, t)=\sum_{n=-\infty}^{\infty} \hat{\varphi}_{n}(x, y) \exp \left(i n \beta z-n \omega_{p} t\right)
$$

In practice, the frequency of secondary instability is often an order of magnitude higher than that of the primary wave, i.e., $\omega_{s} \gg \omega_{p}$, therefore, we consider a secondary instability of a frequency that is an integer multiple of the primary wave frequency for illustrative purpose. Let such a fixed integer be denoted by, $k$, then Eq. (8) becomes

$$
\varphi(x, y, z, t)=\sum_{n=-\infty}^{\infty} \hat{\varphi}_{n}(x, y) \exp \left(i n \beta z-i(n+k) \omega_{p} t\right),
$$

Let us now consider the structure of both the base flow and the secondary fluctuations in the discrete Fourier space $(m, n)$, where $m$ denotes the temporal harmonic and $n$ represents the spanwise harmonic. In the spanwise traveling frame, the base flow is spread over the vertical axis corresponding to Fourier modes $(0, n)$; and the secondary instability mode is spread over the parallel line corresponding to Fourier modes $(1, n)$. In a fixed frame of reference, on the other hand, the same two fields occupy the diagonal lines corresponding to Fourier modes $(n, n)$ and $(n+k, n)$, respectively. Consequently, when the primary instability corresponds to traveling crossflow modes, a linear secondary instability mode in the fixed frame of reference is characterized by multiple frequencies that overlap with the frequency range of the nonlinear primary mode. Therefore, in practice, a fixed probe may not be able to distinguish the contributions of the secondary instability from those of the primary instabilities within the Fourier space. Figure 1(a) illustrates the locations of the primary and secondary modes with distinct frequencies in the discrete Fourier space in the traveling frame of reference. Figure 1(b) shows the corresponding locations in a fixed frame of reference for an illustrative case with $k=9$, wherein the overlap in frequencies within the fixed frame is easily observed.

\section{Flow Configuration and Analysis Codes}

The flow configuration employed in the present study corresponds to the laminar flow airfoil TAMU-003T75(v.90) designed at the Texas A \& M University by Belisle et al ${ }^{16}$ The 9.3 percent thick, 30-degree swept wing is designed to achieve natural laminar flow over approximately 60 and 50 percent of the suction and pressure surfaces, respectively, at the design condition of $M=0.75$, AoA $=0$ deg., and $R e_{c} \approx 17$ million. Here, the nondimensional parameters $M$ and $R e_{c}$ are based on the freestream speed, a streamwise chord length of $c_{s}=3.6576 \mathrm{~m}$ (12 ft), and a freestream temperature of $216.67 \mathrm{deg}$. K (390 deg. R), corresponding to a flight altitude of 12,192 $\mathrm{m}(40,000 \mathrm{ft})$. The chord length normal to the leading edge is then given by $c=c_{s} \cos \left(30^{\circ}\right)=3.1676 \mathrm{~m}$. Design constraints for the wing included (i) a lift coefficient that is typical of subsonic transports, and (ii) a wing thickness distribution that is suitable for a mid-size business jet. Both Tollmien-Schlichting and crossflow instabilities are sufficiently weak at the design angle of attack, so that natural laminar flow should be achievable over a significant portion of the airfoil surface on both suction $(0<x / c<0.6)$ and pressure $(0<x / c<0.5)$ sides without having to employ any external means of boundary layer control. In the present paper, we focus on the 
off design condition corresponding to an angle of incidence equal to -1 degree. At this condition, the crossflow instability on the suction side is even stronger than that at the design condition, ${ }^{17}$ which makes it well suited as a baseline for future investigations of crossflow control.

The mean boundary-layer flow over the suction surface of the airfoil is computed with a boundary layer solver ${ }^{18}$ by using the infinite span approximation in conjunction with the inviscid surface pressure distribution derived from an Euler solution under free flight conditions. ${ }^{16}$ Linear and nonlinear development of the instability modes is computed using parabolized stability equations (PSE) as implemented in the Langley Stability and Transition Analysis Codes (LASTRAC). ${ }^{19}$ For details of the computational methodology employed for linear secondary instability analysis of the traveling crossflow mode, the reader is deferred to the earlier work by Li et al. ${ }^{12}$ As described in Section II, the main difference between the primary and secondary stability analyses is that the basic state for the secondary modes (i.e., the mean boundary layer flow modified by the primary crossflow mode) varies in both surface normal and spanwise directions; and, hence, the instability characteristics of the secondary modes must be analyzed using a planar, partial differential equation based eigenvalue problem, ${ }^{20}$ rather than as an ordinary differential equation based eigenvalue problem of the classical analysis.

DNS computations are performed for both finite amplitude traveling crossflow vortices and their secondary instability. Details of the DNS method used in the analysis can be found in the paper by Martin et al. ${ }^{21}$ Additional details of the DNS methodology are also given in Refs. 1 and 2, wherein the laminar breakdown mechanisms associated with different types of secondary instability modes of the stationary crossflow vortices were investigated for the same TAMU-003T-75(v.90) swept wing configuration. The DNS code solves the compressible Navier-Stokes equations by using generalized curvilinear coordinates. The working fluid is assumed to be an ideal gas with linear Newtonian strain-stress relation. Fourier law is used to compute the heat flux terms. Temperature variation of viscosity is assumed to be given by the Sutherland law and the Prandtl number is assumed to be constant at 0.72 . DNS are performed using both a conventional, stationary frame and a traveling frame that moves in the spanwise direction with the same speed as the component of the phase velocity of the traveling crossflow mode along the span. As discussed in Section II and by Li et al. ${ }^{12}$ the traveling frame has certain advantages for the analysis of secondary instabilities and that simulation is used for comparison with the simulation in the stationary frame. The simulation results pertaining to laminar-turbulent transition are based on the simulation in the traveling frame and a baseline computational grid corresponding to $7380 \times 96 \times 350$ points in the chordwise, spanwise, and wall-normal directions, respectively. In terms of wall units of the fully turbulent flow near the outflow of the domain, the grid resolution in the streamwise $(x)$ and spanwise directions (z) corresponds to $\Delta x^{+} \approx 10$ and $\Delta z^{+} \approx 12.5$, respectively. The first grid point in the wall normal direction is located at $\Delta y^{+} \approx 0.6$. Additional simulations have been performed using a denser grid ( 240 points) in the spanwise direction. However, the length of this simulation is not long enough to permit statistically converged estimates of the turbulent statistics. At present, the latter simulation has only been used to assess the modal evolution ahead of transition onset in the baseline simulation. Computations have also been performed on a smaller domain in streamwise and wall-normal directions that was discretized with a $4350 \times 240 \times 285$ mesh. Following Jiang et al., ${ }^{22}$ the $i$-coordinate of the grid is aligned with the constant phase surfaces of the traveling crossflow vortex to reduce the number of points required to resolve the wavy structure of the mode in the chordwise direction. The computational domain for the stationary frame simulation extends over more than 35 wavelengths of the secondary instability wave introduced at the inflow location.

A $7^{\text {th }}$ order WENO scheme ${ }^{23,24}$ is used to compute the convective flux terms within the DNS code. This particular WENO scheme combines a high order of accuracy with relatively low dissipation, making it a robust option for the simulations of compressible transitional flows, especially when the resolution requirements are not fully known ahead of a simulation. The resolution properties of the WENO scheme used herein are documented in many references, e.g., Martin et al. ${ }^{18}$ For the viscous flux terms, a $4^{\text {th }}$ order central difference scheme is used and the $3^{\text {rd }}$ order low storage Runge-Kutta scheme by Williamson ${ }^{25}$ is employed for time integration. Unsteady non-reflecting boundary conditions based on Thompson ${ }^{26}$ are imposed at the freestream boundary. A sponge region is used near the inflow and freestream boundaries to prevent acoustic reflections to the interior of the domain. ${ }^{27}$ Near the outflow boundary, the computational grid is stretched over an extended region before the primitive variables are extrapolated at the outflow plane. ${ }^{1,2}$ The DNS code has been extensively tested for both fully turbulent ${ }^{28,29}$ and transitional flows ${ }^{1,2}$ in previous work. Convergence studies (not described here) have shown that the $7^{\text {th }}$ order WENO performs well at a resolution of 10 points per wavelength or higher, depending on the streamwise extent of the computational domain. 


\section{Numerical Results}

This section first presents an overview of the linear and nonlinear evolution of the traveling crossflow instability on the TAMU-003T-75(v.90) swept wing configuration, followed by a brief description of the linear secondary instability characteristics. Illustrative results based on the DNS of the nonlinear evolution of a single secondary instability mode are presented thereafter and form the main content of this section.

$\mathrm{Li}$ et al. ${ }^{12}$ used linear PSE to predict the linear $\mathrm{N}$-factor evolution of traveling crossflow modes over a range of disturbance frequencies and spanwise wavelengths. Traveling crossflow instability modes with spanwise wavelengths of $\lambda=6,8$ and $10 \mathrm{~mm}$ and a frequency near $f=1,500 \mathrm{~Hz}$ were found to reach $\mathrm{N}=10$ before most other traveling modes. Since $\lambda=8 \mathrm{~mm}$ was also used in the earlier simulations involving stationary crossflow instability, ${ }^{1,2}$ a traveling mode with $f=1,500 \mathrm{~Hz}$ and $\lambda=8 \mathrm{~mm}$ was chosen as the primary instability during the present DNS of secondary instability and subsequent breakdown to a turbulent flow. The above traveling mode reaches $\mathrm{N}=10$ within approximately half the distance it takes for the stationary crossflow mode $(f=0)$ to reach the same $\mathrm{N}$-factor via linear amplification.

Comparisons between the nonlinear development of traveling and stationary vortices were presented by Choudhari et al. ${ }^{2}$ Their findings showed that the traveling crossflow vortices at $f=1,500 \mathrm{~Hz}$ reach a quasisaturation stage at lower fundamental amplitudes than the stationary vortices, specifically at approximately just one half of the saturation amplitude of the stationary modes. On the other hand, the peak mean flow correction amplitudes for both stationary and traveling modes were found to be comparable to each other. These features of the nonlinear evolution of isolated traveling and stationary crossflow disturbances are similar to those reported by Malik et al. ${ }^{30}$ for a different airfoil configuration as well as to the findings of Wassermann and Kloker ${ }^{8}$ for a boundary-layer flow past a flat plate that is subjected to a favorable pressure gradient. NPSE computations of traveling crossflow mode ${ }^{2}$ also showed that nonlinearity caused the amplitude of the fundamental mode to evolve in an oscillatory manner following the initial rise to the first peak, the latter representing the global maximum of the fundamental mode amplitude. The oscillatory behavior was superimposed on a decaying trend and terminated with a premature decay well upstream of the upper branch neutral location predicted by the linear theory. ${ }^{2,12}$

To help validate NPSE predictions for nonlinear evolution of the primary, traveling crossflow instabilities, DNS computations were also carried out for $1,500 \mathrm{~Hz}$ mode by using the traveling frame of reference discussed in Section II. For this primary-only simulation, a smaller spatial grid of size $981 \times 40 \times 278$ was used in the streamwise, spanwise and wall-normal directions, respectively. To save on the computational resources, the chordwise extent of the domain is restricted by choosing an inflow location at $x / c=0.15$, i.e., somewhat farther downstream from the lower branch neutral station at $x / c \approx 0.05$. The linear eigenfunction of the $1,500 \mathrm{~Hz}$ traveling crossflow mode is imposed at the inflow with an initial amplitude that is equivalent to $A_{\text {init }}=5 \times 10^{-7}$. The DNS results are compared with the NPSE results in Figure 2 with $\mathrm{m}$ denoting the index of the temporal harmonic. The amplitude evolution of the fundamental mode $(m=1)$ from DNS compares very well with that from NPSE right from the beginning of the DNS domain. This is not the case for the mean flow correction mode and the higher harmonics. For DNS, these modes undergo a transient phase, but eventually settle to match the NPSE predictions.

As shown previously, ${ }^{2,12}$ the quasi-saturated, finite amplitude traveling crossflow mode can support rapidly growing secondary instabilities ${ }^{12}$ in much the same way as the stationary crossflow modes. ${ }^{31}$ Despite the lower amplitudes during the quasi-saturation stage, computational predictions for the configuration of interest have shown that the growth of secondary instabilities sustained by the traveling crossflow vortices can be as strong as that of the secondary modes of stationary crossflow vortices. The secondary instability of the non-stationary crossflow vortex with $\lambda=8 \mathrm{~mm}$ and $f=1,500 \mathrm{~Hz}$ was discussed in Refs. 2 and 12 . Typically, two types of secondary instability modes were observed in this case, namely, a Y mode that primarily derives its energy production mechanism from the wall-normal shear of the modified basic state and a Y/Z mode that is associated with both wall-normal and spanwise shear of the basic state. The Y mode dominates at sufficiently high initial amplitudes of the traveling crossflow mode (at approximately $A_{\text {init }} \geq 5 \times 10^{-6}$, where the non-dimensional amplitude $A_{\text {init }}$ for the primary disturbances is defined as the ratio of the peak chordwise perturbation velocity at the lower branch neutral station to the freestream speed). As the primary amplitude decreases, the peak $\mathrm{N}$-factor of the Y mode decreases rather rapidly and, hence, the Y/Z mode has the largest linear amplification ratios for lower initial primary amplitudes. At the initial primary amplitude of $2.5 \times 10^{-6}$, the peak Y-mode $\mathrm{N}$-factor is approximately 13 and the $\mathrm{Y} / \mathrm{Z}$-mode $\mathrm{N}$-factor has already overtaken the $\mathrm{Y}$ mode with a peak value of 18 . Upon further reduction of the initial primary wave amplitude to $5 \times 10^{-7}$, no Y mode is found by the LSIT code, indicating that the Y-mode secondary instability has become substantially weaker. On the other hand, the Y/Z 
mode of secondary instability still reaches a peak $\mathrm{N}$-factor of approximately 10 . Because lower initial amplitudes of traveling crossflow modes are deemed to be more relevant to aircraft flight due to relatively quiet ambient conditions, the case of $A_{\text {init }}=5 \times 10^{-7}$ is chosen for the numerical simulations described in this paper. DNS results for the nonlinear development of secondary instability and the subsequent breakdown to turbulence are presented in Subsections A and B below.

\section{A. Nonlinear Evolution of Secondary Instability}

The effect of initial disturbance amplitude on the secondary instability evolution is addressed in this section as a precursor to the simulations of laminar-turbulent transition. To that end, numerical simulations were carried out using the traveling frame of reference for a fixed-frequency secondary instability mode with a frequency of $13.5 \mathrm{kHz}$, which is nine times larger than the frequency of the traveling crossflow mode. The integer ratio of secondary to primary disturbance frequencies for the traveling frame computations is purely for the convenience of post-processing during analogous simulations in a stationary frame. The selected frequency of $13.5 \mathrm{kHz}$ is close to the most amplified $\mathrm{Y} / \mathrm{Z}$ mode for the chosen case of primary, traveling crossflow mode with $\lambda=8 \mathrm{~mm}, f$ $=1,500 \mathrm{~Hz}$, and $A_{\text {init }}=5 \times 10^{-7}$. As discussed before, at the relatively low value of $A_{\text {init }}$ for the primary crossflow vortex, the $\mathrm{Y}$ mode of secondary instability is nearly stable, but the $\mathrm{Y} / \mathrm{Z}$ mode is still strong. The linear eigenfunction of the $13.5 \mathrm{kHz}$ secondary instability mode (Fig. 3) is imposed at an inflow location of $x / c \approx 0.301$, which is somewhat downstream of the neutral point where this mode first begins to amplify. The minimum wavelength of this mode in the $i$-direction of the grid is approximately $12.7 \mathrm{~mm}$. No additional, random disturbances are introduced in these computations to induce smaller scale structures, which are allowed to emerge on their own, either via a cascade of nonlinear interactions or from the discretization errors.

Figure 4 shows the amplitude evolution of the $13.5 \mathrm{kHz}$ Y/Z-mode secondary instability for four different initial amplitudes. In each case, the secondary wave amplitude is normalized by its initial value, so that the ordinate in this figure corresponds to the growth factor relative to the inflow location. The evolution of actual (i.e., unscaled) fundamental amplitudes for these cases is shown in Fig. 5. Figs. 4 and 5(a) show that, for the lowest initial amplitude $\left(A_{\text {init }}=1.76 \times 10^{-7}\right)$, the evolution of the secondary disturbance remains linear throughout the computational domain. Although predictions based on either secondary instability theory or PSE computations for this low initial amplitude are not shown in the figure, they both agree well with the DNS results shown here. For the two intermediate initial amplitudes, weakly nonlinear effects come into play after the secondary amplitude becomes sufficiently large $(x / c>0.38)$ and the growth of the fundamental secondary disturbance becomes slower than that for the nominally linear case of $A_{\text {init }}=1.76 \times 10^{-7}$. Eventually, however, the secondary disturbance decays without indicating any sign of transition onset. The NPSE predictions for $A_{\text {init }}=$ $9.5 \times 10^{-4}$ at $x / c \approx 0.301$ (which corresponds to the same effective amplitude at the lower branch of the secondary instability at the DNS case with $A_{\text {init }}=8.8 \times 10^{-4}$ at $\left.x / c \approx 0.301\right)$ are also indicated using symbols in this figure and they agree well with the DNS result. A similar growth behavior is observed when the initial secondary amplitude is increased to $0.206 \%$. However, the saturation location shifts further upstream and is now within the range of streamwise locations plotted in the figure. At the highest initial amplitude of the secondary mode $\left(A_{\text {init }}\right.$ $=1.00 \times 10^{-2}$ at $x / c \approx 0.301$ ), the amplitude curve becomes nearly flat within the region of quasi-saturation; however, rather than decaying farther downstream, it actually increases again to a second plateau before undergoing a rapid growth near $x / c=0.39$. As seen later, this location approximately coincides with the onset of transition. The rapid growth in fundamental amplitude, corresponding to a rise in peak amplitude from approximately $13 \%$ to $17 \%$ (see Fig. 5(b)), is followed by a nearly exponential decay across the transition region before the amplitude levels appear to level off just upstream of $x / c=0.41$. Farther downstream, the fundamental amplitude exhibits a random yet bounded variation within the range of approximately $6 \%-8 \%$, suggestive of a stochastic disturbance field corresponding to turbulent boundary-layer flow.

A comparison of DNS and PSE predictions for disturbance amplitudes at the fundamental frequency (Mode 1) of the secondary disturbance and its selected harmonics (Mode $>1$ ) for an initial secondary amplitude of $1.00 \times 10^{-2}$ are shown in Fig. 6. DNS results based on both the baseline grid and shorter duration simulations based on the refined grid with 240 spanwise points are included in the figure. The two sets of DNS predictions agree well throughout the deterministic portion of secondary evolution, i.e., up to and somewhat beyond the onset of transition. The fundamental evolution based on the PSE computation (which was terminated somewhat ahead of the transition onset near $x / c=0.39$ ) agrees well with the DNS results up to $x / c=0.375$, but noticeable deviations are seen for the higher harmonics excited via nonlinear interactions. Further work to resolve the source of this discrepancy is currently ongoing. 
In the spanwise traveling frame employed for the DNS (and PSE) computations in Figs. 5 and 6, the base flow is purely stationary, i.e., it corresponds to Fourier modes $(0, n)$ in the frequency-spanwise wavenumber plane where $n=-\infty \rightarrow \infty$, and the fluctuations associated with the linear secondary instability (i.e., the fundamental harmonic of secondary disturbance) correspond to the adjacent vertical line corresponding to Fourier modes $(1, n)$. In a fixed frame of reference, on the other hand, the base flow and fundamental secondary mode occupy the diagonal lines corresponding to Fourier modes $(n, n)$ and $(n+k, n)$, respectively, where $k=9$ denotes the ratio of secondary mode frequency in the traveling frame of reference and the frequency of the primary crossflow mode. Consequently, a linear secondary instability mode in the fixed frame of reference is characterized by multiple frequencies that overlap with the frequency range of the nonlinear primary mode. Therefore, in practice, a fixed probe may not be able to distinguish the contributions of the secondary instability from those of the primary instabilities within the Fourier space. Therefore, the traveling frame simulations are better suited for the analysis of weak to moderately nonlinear effects on the secondary mode evolution, but the stationary frame is more appropriate for the broad-band fluctuations fields induced by the breakdown process that one might wish to measure in an experiment using conventional instrumentation. The latter point is illustrated using a stationary-frame simulation for $A_{\text {init }}=1 \%$. The chordwise evolution of secondary mode amplitudes is plotted in Fig. 7.

The disturbance evolution in the stationary frame (Fig. 7) is considerably more complex than the traveling frame results illustrated in previous figures. This is to be expected because a single mode of secondary instability consists of multiple frequencies as discussed above and, therefore, each temporal harmonic in Fig. 7 includes contributions from both primary and secondary disturbances. Close to the inflow $(x / c \approx 0.301)$, the lower temporal harmonics (especially modes labeled as 1 and 2) are dominated by the primary, traveling crossflow mode because of the initially smaller amplitudes of the secondary disturbance. Hence, the corresponding red curves indicating the mixed evolution of primary and secondary disturbances nearly overlap in the upstream portion of the plot. Harmonics 6 and 5 indicate the strongest signature of the secondary disturbance in the upstream region and they indicate a nearly exponential growth in that region (barring a short region of apparently transient behavior in mode 6 evolution because of an imperfect match between the mode shape predicted by the secondary instability theory and the DNS solution). As the secondary disturbances gain in amplitude, even the lower temporal harmonics receive an increasingly significant contribution from the secondary disturbance and, hence, the combined modal evolution begins to depart from the primary-only evolution indicated by the corresponding green curves near $x / c=0.33$. Harmonics 5 and 6 associated with the secondary disturbance overtake the predominantly primary fundamental mode (labeled as 1 ) near $x / c=0.345$.

To understand the structural evolution of the flow in the stationary frame, Fig. 8 indicates an instantaneous snapshot of the flowfield at a selected instant of time. Crossplane visualizations of instantaneous chordwise velocity contours at successively downstream chordwise stations are shown in Figs. 8(a) through 8(d). At the inflow station $(x / c \approx 0.301)$, the crossplane contours are dominated by the fundamental spanwise harmonic corresponding to the traveling crossflow mode. However, the contours corresponding to the highest shear away from the wall indicate three smaller and approximately uniformly spaced lobes for chordwise locations between $x / c=0.32$ and $x / c=0.36$ and as many as four smaller lobes may be noted at $x / c=0.37$. These lobes are associated with the secondary disturbances. In particular, the most energetic $n=6$ temporal harmonic from Fig. 2(b) corresponds to a spanwise wavenumber of three times the wavenumber of the traveling crossflow mode and the $n=5$ temporal harmonic represents a wavenumber that is four times the primary wavenumber.

The role of secondary disturbances in inducing the shorter scale spanwise disturbances that can presumably bring about transition is easily seen by comparing the velocity contours for joint primary and secondary evolution (Fig. 8) with those for the primary-only evolution (i.e., without any secondary disturbance) in Fig. 9. The latter contours remain dominated by the fundamental primary wavelength throughout the region of interest; and, hence, no onset of transition is likely to be observed in the presence of purely primary disturbances.

\section{B. Breakdown of Secondary Instability}

The traveling frame simulations in Figs. 4 through 6 showed that transition occurs when the initial amplitude of the secondary disturbance has reached a sufficiently large value (bracketed by $A_{\text {init }}=0.206 \%$ and $A_{\text {init }}=1 \%$ ). The modal evolution in Fig. 6 indicates that the onset of transition is immediately preceded by a rapid rise in the amplitudes of the higher harmonics of the secondary disturbance. The decay of the fundamental harmonic across the transition zone as well as the region of fully developed turbulence is accompanied by an analogous decay in these higher harmonics. 
To further understand the evolution of the flow through the transition process, Fig. 10 displays the evolution of (short duration) RMS fluctuations in surface tangential velocity across selected chordwise stations ranging from ahead of transition onset (Fig. 10(a)), within the transition zone (Fig. 10(b)) and the fully turbulent region (Fig. 10(c)). The initial evolution in Fig. 10(a) is qualitatively analogous to the mode shapes of secondary instability within the moving frame (recall Fig. 3). The peak of these mode shapes occurs at a finite distance from the airfoil surface, near the location of the internal, inclined shear layer associated with a finite amplitude traveling crossflow mode. As the secondary instability becomes stronger, this inclined shear layer develops spanwise oscillations (not unlike the structures observed in the context of Z-mode secondary instability of stationary crossflow modes in Ref. 2). The strengthening of the spanwise oscillations leads to multiple islands of peak fluctuation amplitudes. Transition onset appears to coincide with the location when one or more of these peaks approach the wall, wetting the surface with high intensity fluctuations within a narrow range of spanwise locations. As transition proceeds, additional peaks are observed in the vicinity of the surface, eventually filling up the entire spanwise region. Concomitantly, the signature of the inclined shear layer fades away from the (short time) averaged mean velocity contours, which resemble a simpler sinusoidal undulation apparently associated with the remnant of the fundamental mode of the primary crossflow instability. These structures appear stationary within the moving frame, but translate in the spanwise direction when viewed within a stationary frame. Consequently, the mean flow becomes homogeneous along the spanwise direction in the present case of traveling crossflow breakdown.

The evolution of mean wall shear associated with the chordwise velocity profile is plotted in Fig. 11. Wall shear contours along the surface (Fig. 11(a)) indicate that the onset of high shear (symptomatic of transition) is initially concentrated at a specific spanwise location within the traveling frame and that the turbulence spreads in approximately wedge shaped regions until the adjacent wedge shaped regions merge with each other and the skin friction reaches turbulent values throughout the span. Of course, in a stationary frame, the wedges sweep across the span at the phase velocity of the primary disturbance; and, hence, the mean wall shear evolves uniformly at all spanwise locations as shown in Fig. 11(b). In other words, the transition front is nominally smooth in the spanwise direction and does not resemble the sawtooth pattern that is commonly seen in naphthalene based surface flow visualizations of crossflow transition dominated by stationary vortices. Experimental flow visualizations have confirmed that the transition front associated with traveling crossflow disturbances is indeed smooth in nature. ${ }^{30}$ The mean shear evolution in Fig. 11(b) indicates a rapid rise in the near-wall mean velocity at $x / c \approx 0.39$, indicating the onset of transition location. The skin friction reaches a peak near $x / c=0.41$ and then decreases weakly over the remaining length of the computational domain.

Finally, the instantaneous flow visualization in Fig. 12 shows how the flow structures evolve across the transition region. The pre-breakdown, nonlinear secondary instability manifests itself as corrugations over the tubular isosurface representing the traveling crossflow vortex within the moving frame. These corrugated structures are analogous to those observed in the context of secondary instability modes of the stationary crossflow vortices in Refs. 1 and 2. The main difference, however, is that the secondary corrgugations associated with the traveling modes are inclined at a shallower angle with respect to the axis of the underlying primary vortex.

\section{Concluding Remarks}

This paper has extended the previously reported computational studies of swept wing transition due to stationary crossflow instability by considering the laminar breakdown of traveling crossflow modes due to highfrequency secondary instability. As discussed in Refs. 2 and 12, the traveling crossflow vortices over swept wing boundary layers support secondary instabilities in much the same way as the stationary crossflow modes. Herein, we considered the canonical case involving a single traveling crossflow mode (with $\lambda=8 \mathrm{~mm}, f=1,500$ $\mathrm{Hz}$, and $A_{\text {init }}=5 \times 10^{-7}$ ) along with a single mode of secondary instability. The secondary mode considered herein corresponds to the most amplified secondary disturbance $(f=13,500 \mathrm{~Hz}$ in the traveling frame $)$ and is of the $\mathrm{Y} / \mathrm{Z}$ type, i.e., its energy production mechanisms involve comparable contributions from both wall-normal and spanwise shears associated with the modified basic state corresponding to a superposition of the mean boundary layer flow and a finite amplitude traveling crossflow mode. DNS computations show that transition onset will occur when the initial amplitude of the secondary instability mode exceeds a threshold value that lies between $A_{\text {init }}=2.06 \times 10^{-3}$ and $A_{\text {init }}=1.0 \times 10^{-2}$ at $x / c=0.301$.

Direct numerical simulations are performed for a $1 \%$ initial amplitude of the secondary instability mode using a spanwise-traveling coordinate frame and the transition onset is observed to occur near $x / c=0.39$. The 
peak fundamental secondary disturbance amplitude just before transition is approximately $15 \%$ of the freestream speed. Simulation results confirm that the transition front in a stationary frame would be homogeneous along the spanwise direction, i.e., would not display the sawtooth pattern observed in both experiments and numerical simulations of the breakdown of stationary crossflow vortices. Nonetheless, transition occurs rapidly similar to the case of stationary crossflow breakdown and the width of the transition zone is less than a distance of approximately fifteen times the boundary layer thickness at the location of fully developed turbulence. The length of the transition zone appears to be mainly determined by the traveling crossflow wavelength and the turbulence spreading angle. Alternately, the relatively short length of the transition region also suggests that modeling transition as an instantaneous process could provide reasonable accuracy for RANS based prediction in the present case.

\section{Acknowledgments}

This work was performed as part of the Revolutionary Computational Aerosciences (RCA) discipline under the Aeronautical Sciences (AS) project of NASA's Fundamental Aeronautics Program. The authors would like to thank Prof. Pino Martin of the University of Maryland for providing the original version of the DNS code used in this effort.

\section{References}

1 Duan, L., Choudhari, M., and Li, F., "Direct Numerical Simulation of Crossflow-Induced Transition in a Swept Wing Boundary Layer,” AIAA Paper 2013-2617, 2013.

2 Choudhari, M., Li, F., Duan, L., Carpenter, M. H., Streett, C. L., and Malik, M. R., "Towards Bridging the Gap in Hoslistic Transtion Prediction via Numerical Simulations," AIAA Paper 2013-2718, 2013.

${ }^{3}$ Poll, D. I. A., "Transition in the Infinite Swept Attachment Line Boundary Layer," The Aeron. Quarterly, Vol. 30, 1979, pp. 607-629.

${ }^{4}$ Malik, M. R., Li, F., Choudhari, M., and Chang, C.-L., "Secondary Instability of Crossflow Vortices and Swept-Wing Boundary-Layer Transition,” J. Fluid Mech., Vol. 399, 1999, pp. 85-115.

${ }^{5}$ Kohama, Y., Saric, W. S., and Hoos, J. A., "A high-frequency secondary instability of crossflow vortices that leads to transition," in Boundary Layer Transition and Control, Proc. Royal Aeronautical Society, Cambridge, 8-12 April, 1991, London, UK.

${ }^{6}$ Balachandar, S., Streett, C. L., and Malik, M. R., "Secondary Instability in a Rotating Disk Flow," J. Fluid Mech., Vol. 242, 1992, pp. 323-347.

${ }^{7}$ Malik, M. R., Li, F., and Chang, C.-L., "Crossflow disturbances in three-dimensional boundary layers: Nonlinear development, wave interaction and secondary instability," J. Fluid Mech., Vol. 268, 1994, pp. 1-36.

${ }^{8}$ Wassermann, P. and Kloker, M., "Mechanisms and Passive Control of Crossflow-Vortex-Induced Transition in a Three-Dimensional Boundary Layer," J. Fluid Mech., Vol. 456, 2002, pp. 49-84.

${ }^{9}$ Bippes, H., "Basic experiments on transition in three-dimensional boundary layers dominated by crossflow instability," Progress in Aerospace Sciences, Vol. 35, 1999, pp. 363-412.

${ }^{10}$ Wassermann, P. and Kloker, M., "Transition Mechanisms Induced by Traveling Crossflow Vortices in a Three-Dimensional Boundary Layer," J. Fluid Mech., Vol. 483, 2003, pp. 67-89.

${ }^{11}$ Herbert, T., "Secondary Instability of Boundary Layers," Ann. Rev. Fluid Mech., Vol. 20, 1988, pp. 487-526.

${ }^{12}$ Li, F., Choudhari, M., Duan, L, and Chang, C.-L., "Nonlinear Development and Secondary Instability of Traveling Crossflow Vortices," Physics of Fluids, Vol. 26, 064104, 2014.

${ }^{13}$ Down, R. S. and White, E. B., "Free-stream Turbulence and Development of Crossflow Disturbances," J. Fluid Mech., Vol. 735, 2013, pp. 347-380.

${ }^{14}$ Li, F. and Choudhari, M., "Spatially Developing Secondary Instabilities in Compressible Swept Airfoil Boundary Layers," Theor. Comput. Fluid Dyn., Vol. 25, 2011, pp. 65-84.

${ }^{15}$ Malik, M. R., Li, F., and Chang, C.-L., "Nonlinear Crossflow Disturbances and Secondary Instability in Three-Dimensional Boundary Layers," Proc. IUTAM Symp., Kluwer Acad. Publ., pp 257-266, 1996.

${ }^{16}$ Belisle, M., Neale, T., Reed, H., and Saric, W. "Design of a Swept-Wing Laminar Flow Control Flight Experiment for Transonic Aircraft," AIAA paper 2010-4381, 2010.

${ }^{17}$ Saric, W. S., "Flight Experiments on Swept-Wing Roughness Receptivity: Phase 2. Feasibility of a laminar flow flight test," Phase 2, Year 1 Draft Report for AFOSR Grant FA955008-1-0475, June, 2009.

${ }^{18}$ Wie, Y.-S., "BLSTA - A Boundary Layer Code for Stability Analysis," NASA CR 4481, 1992. 
${ }^{19}$ Chang, C.-L., "Langley Stability and Transition Analysis Code (LASTRAC) Version 1.2 User Manual," NASA/TM-2004-213233, June, 2004.

${ }^{20}$ Lin, R. S. and Malik, M. R., "On the Stability of Attachment-line Boundary Layers. Part I. The Incompressible Swept Hiemenz Flow," J. Fluid Mech., Vol. 311, 1996, pp. 239-255.

${ }^{21}$ Martin, M. P., Taylor, E. M., Wu, M., and Weirs, V. G., "A Bandwidth-Optimized WENO Scheme for Effective Direct Numerical Simulations of Compressible Turbulence," J. Comp. Phys., Vol. 220, 2006, pp. 270 298.

${ }^{22}$ Jiang, L., Choudhari M., Chang, C., and Liu, C., "Direct Numerical Simulations of Crossflow Disturbances in Supersonic Boundary Layers," AIAA Paper 2004-589, 2004.

${ }^{23}$ Jiang, G. S. and Shu, C. W., "Efficient Implementation of Weighted ENO Schemes," J. Comp. Phys., Vol. 126, No. 1, 1996, pp. 202-228.

${ }^{24}$ Taylor, E. M., Wu, M., and Martın, M. P., "Optimization of Nonlinear Error Sources for Weighted NonOscillatory Methods in Direct Numerical Simulations of Compressible Turbulence," J. Comp. Phys., Vol. 223, No. 1, 2006, pp. 384-397.

${ }^{25}$ Williamson, J., "Low-Storage Runge-Kutta Schemes," J. Comp. Phys., Vol. 35, No. 1, 1980, pp. 48.

${ }^{26}$ Thompson, K. W., "Time Dependent Boundary Conditions for Hyperbolic Systems," J. Comp. Phys., Vol. 68, No. 1, Jan. 1987, pp. 1-24.

${ }^{27}$ Duan, L., Choudhari, M., and Li, F., "DNS of Laminar-Turbulent Transition in Swept-Wing Boundary Layers," CTR Research Briefs, 2014.

${ }^{28}$ Duan, L., Beekman, I., and Mart'ın, M. P., "Direct Numerical Simulation of Hypersonic Turbulent Boundary Layers. Part 3: Effect of Mach Number," J. Fluid Mech., Vol. 672, 2011, pp. 245-267.

${ }^{29}$ Duan, L., Choudhari, M., and Wu, M., "Numerical Study of Acoustic Radiation due to a Supersonic Turbulent Boundary Layer,” J. Fluid Mech., Vol. 746, 2013, pp. 165-192.

${ }^{30}$ Malik, M.R., Liao, W., Li, F., and Choudhari, M., "Discrete-Roughness-Element-Enhanced Swept-Wing Natural Laminar Flow at High Reynolds Numbers," AIAA Journal, Mar., 2015. DOI: 10.2514/1.J053637.

${ }^{31}$ White E. B. and Saric W. S., "Secondary Instabilty of Crossflow Vortices," J. Fluid Mech. Vol. 525, 2005, pp. $275-308$.

32 Malik, M. R., Li, F., and Chang, C.-L., "Nonlinear Crossflow Disturbances and Secondary Instability in Three-Dimensional Boundary Layers,” Proc. IUTAM Symp., Kluwer Acad. Publ., pp 257-266, 1996.

${ }_{33}$ Cattafesta, L. N., III, Iyer, V., Masad, J. A, King, R. A., and Dagenhart, J. R., "Three-Dimensional Boundary-Layer Transition on a Swept Wing at Mach 3.5," AIAA Journal, Vol. 33, No. 11, 1995, pp. 2032-2037. 


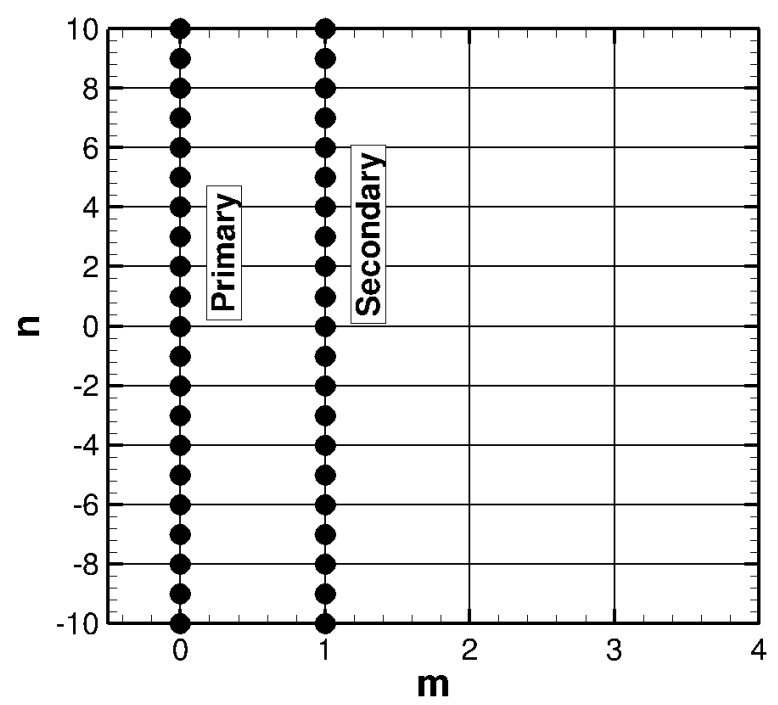

(a) In traveling frame of reference, primary and secondary modes have distinct frequencies.

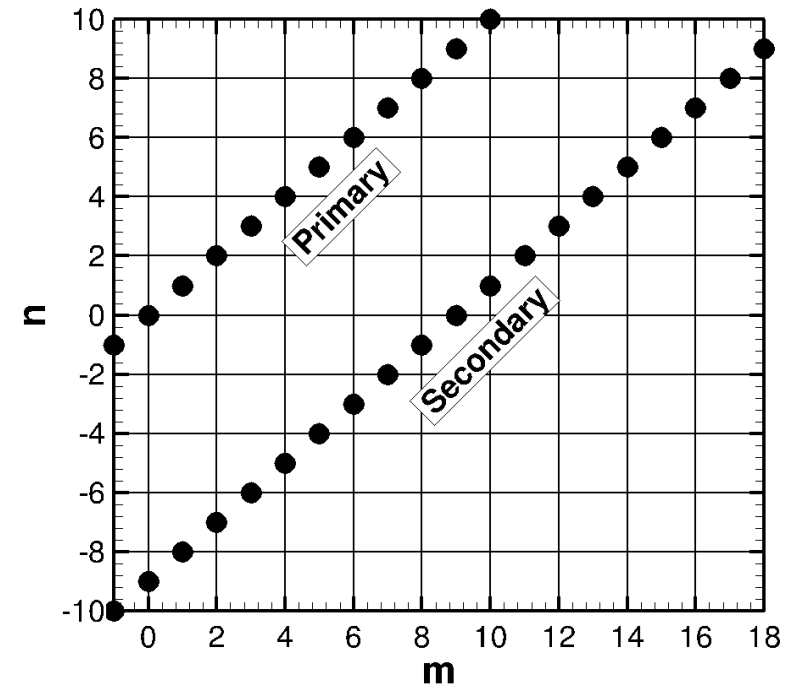

(b) In fixed frame of reference $(k=9)$, primary and secondary modes have overlapping frequencies.

Figure 1. Schematic of primary and secondary modes in discrete Fourier space.

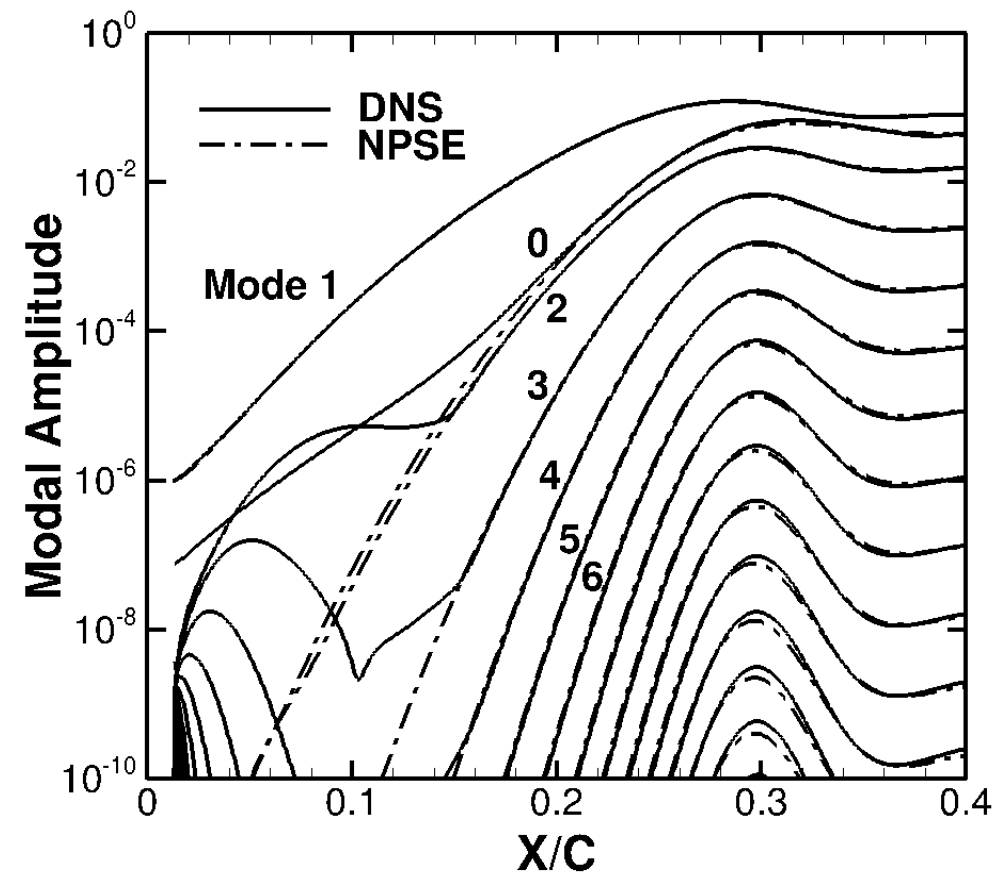

Figure 2. Evolution of modal amplitudes for traveling crossflow vortices with initial amplitude of $5 \times 10^{-7}$. Comparison between results of nonlinear PSE and direct numerical simulations. Solid lines and dash-dot lines present results of DNS and NPSE computations, respectively. 


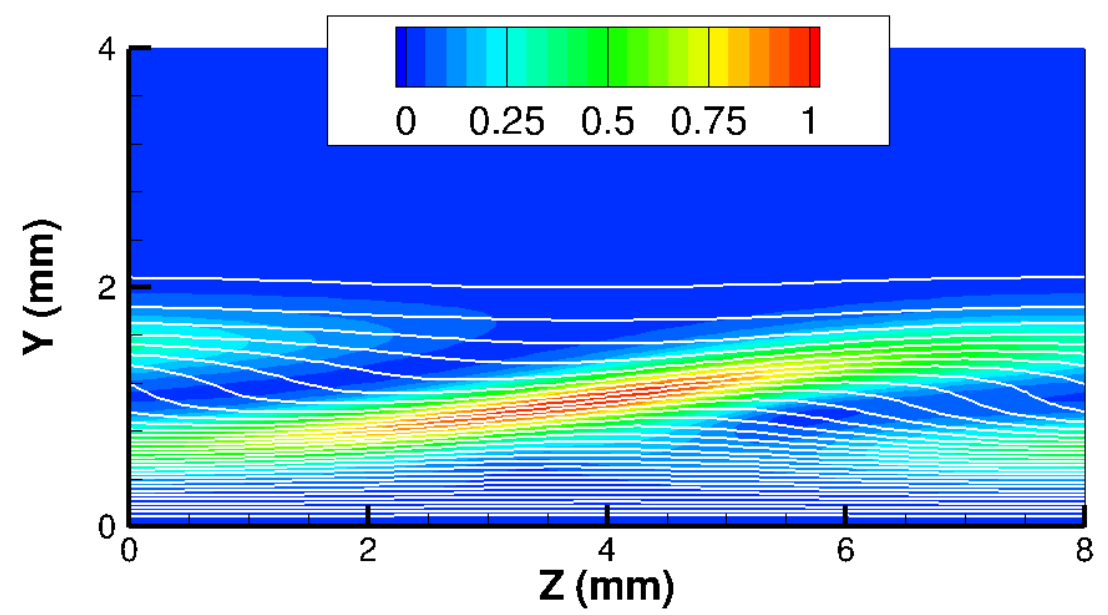

Figure 3. Magnitude of inflow eigenfunction at $x / c \approx 0.301$ for the chordwise velocity perturbation $u$ (normalized to have a peak value of unity over the cross section of the computational domain) associated with a secondary disturbance of type $\mathrm{Y} / \mathrm{Z}$ and a frequency of $13.5 \mathrm{kHz}$ in traveling frame.

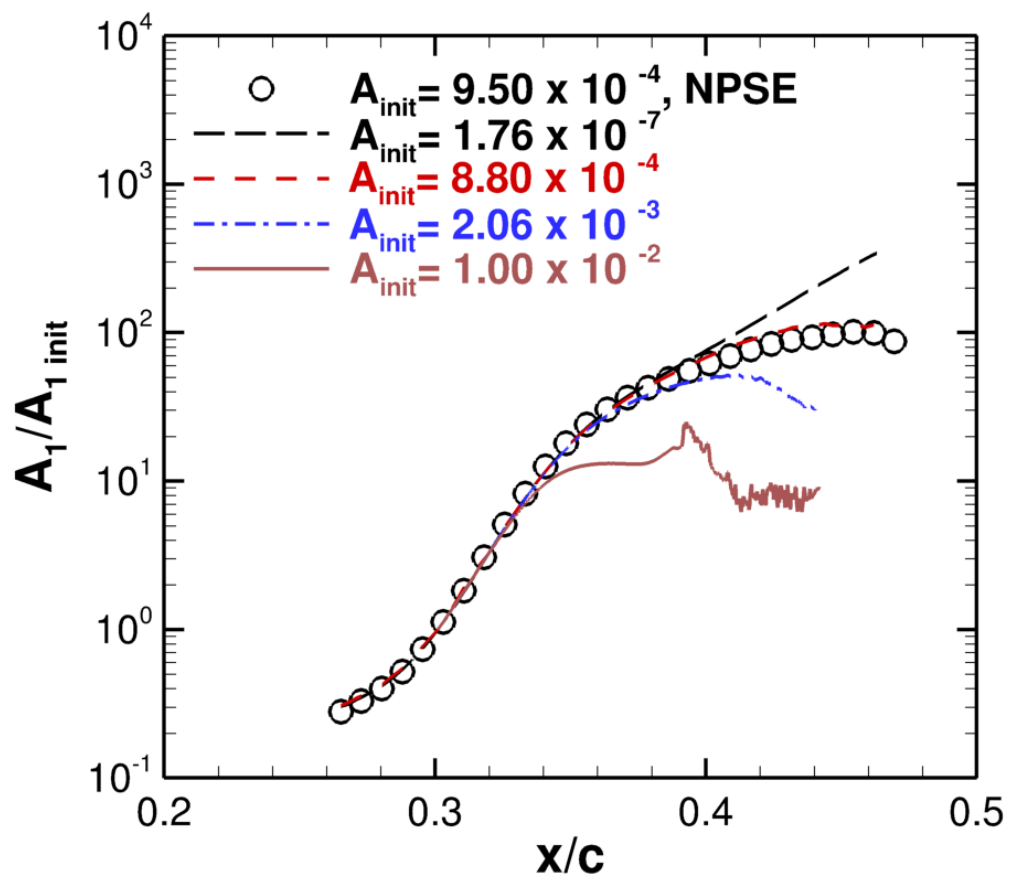

Figure 4. Effect of initial amplitude on chordwise evolution of fundamental secondary mode of type $\mathrm{Y} / \mathrm{Z}$ and a frequency of $13.5 \mathrm{kHz}$ in traveling frame. The primary disturbance parameters correspond to $\lambda=8$ $\mathrm{mm} ; f=1,500 \mathrm{~Hz} ; A_{\text {init }}=5 \times 10^{-7}$. Symbols indicate nonlinear PSE predictions for a secondary initial amplitude of $9.5 \times 10^{-4}$ at the onflow station of $x / c \approx 0.301$. 


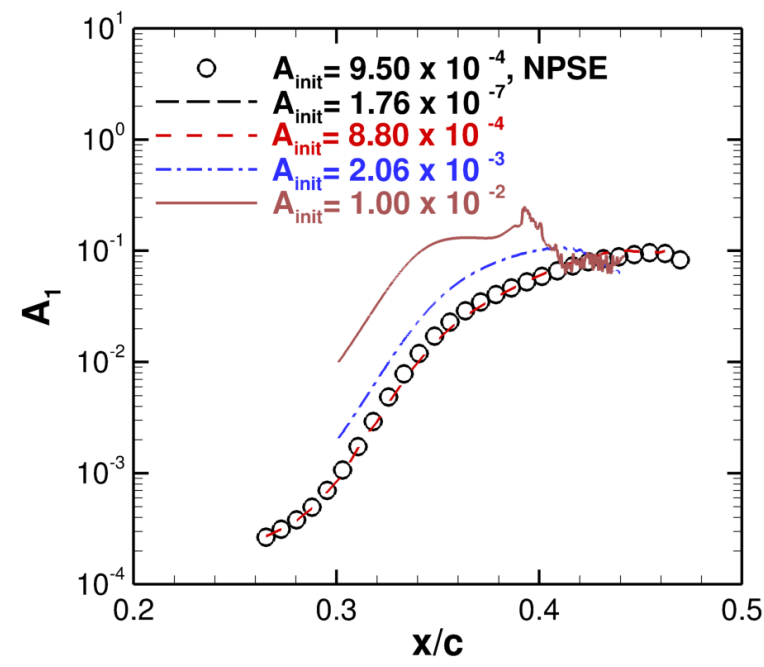

(a) Log-linear axes

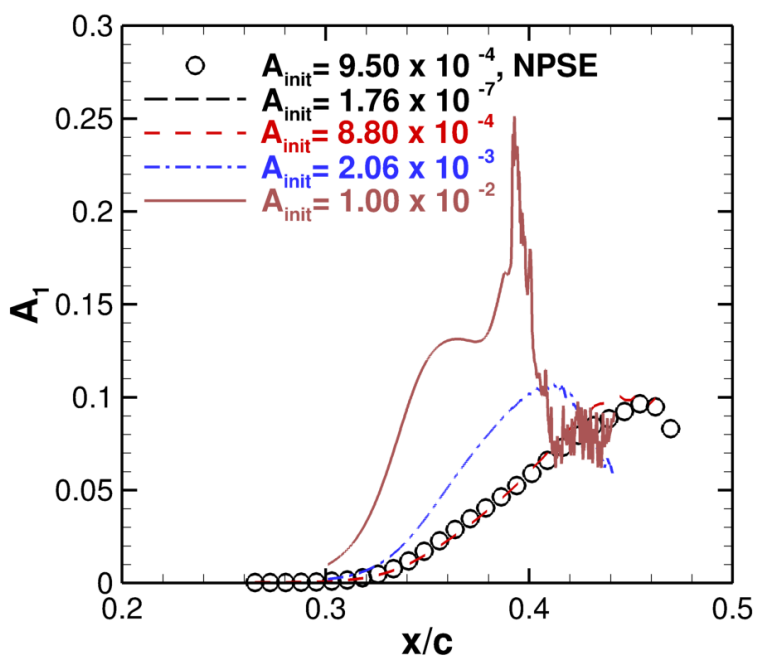

(b) Linear axes

Figure 5. Effect of initial amplitude on chordwise evolution of fundamental secondary mode of type $Y / Z$ and a frequency of $13.5 \mathrm{kHz}$ in traveling frame. The primary disturbance parameters correspond to $\lambda=8$ $\mathrm{mm} ; f=1,500 \mathrm{~Hz} ; A_{\text {init }}=5 \times 10^{-7}$. Symbols indicate nonlinear PSE predictions for a secondary initial amplitude of $9.5 \times 10^{-4}$.

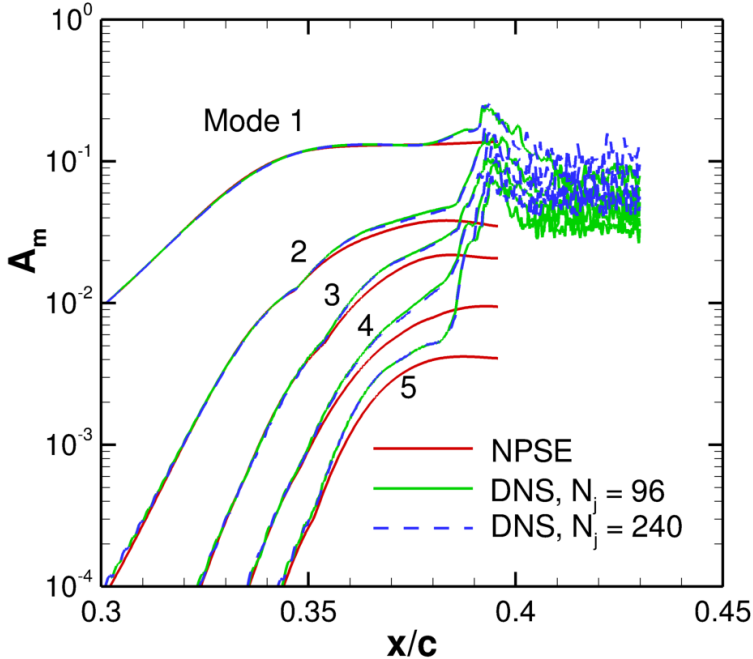

Figure 6. Evolution of disturbance amplitudes at fundamental frequency (Mode 1) of secondary disturbances and its harmonics (Mode > 1) for initial secondary amplitude of $1.03 \%$.

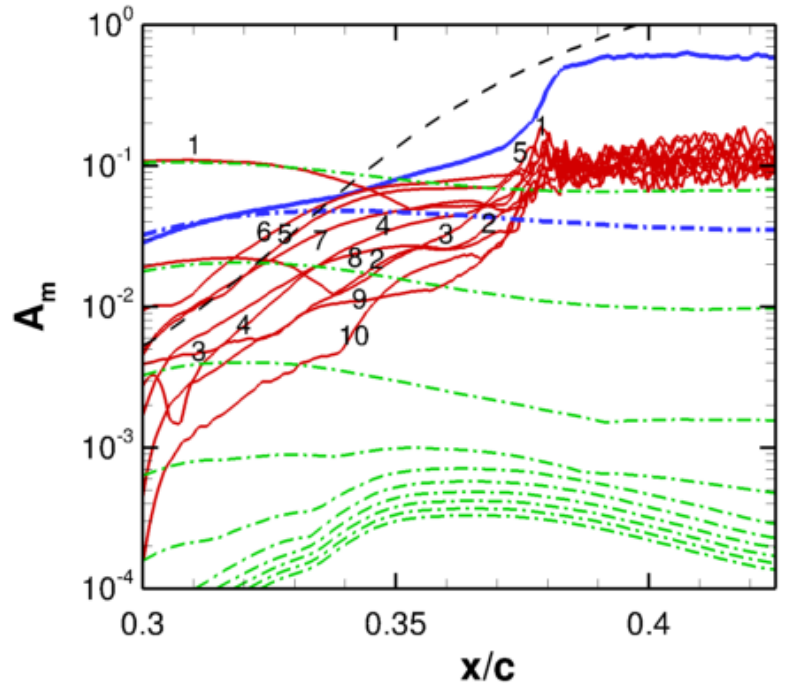

Figure 7. Chordwise variation in peak amplitudes of selected temporal harmonics of unsteady fluctuations in the stationary frame. The red curves indicate the evolution of modal amplitudes corresponding to different harmonics of the frequency corresponding to the primary, traveling crossflow mode. The green curves indicate similar results for primary-only evolution, i.e., in the absence of any secondary instability. The numbered labels indicate the index of temporal harmonic relative to the traveling mode. 


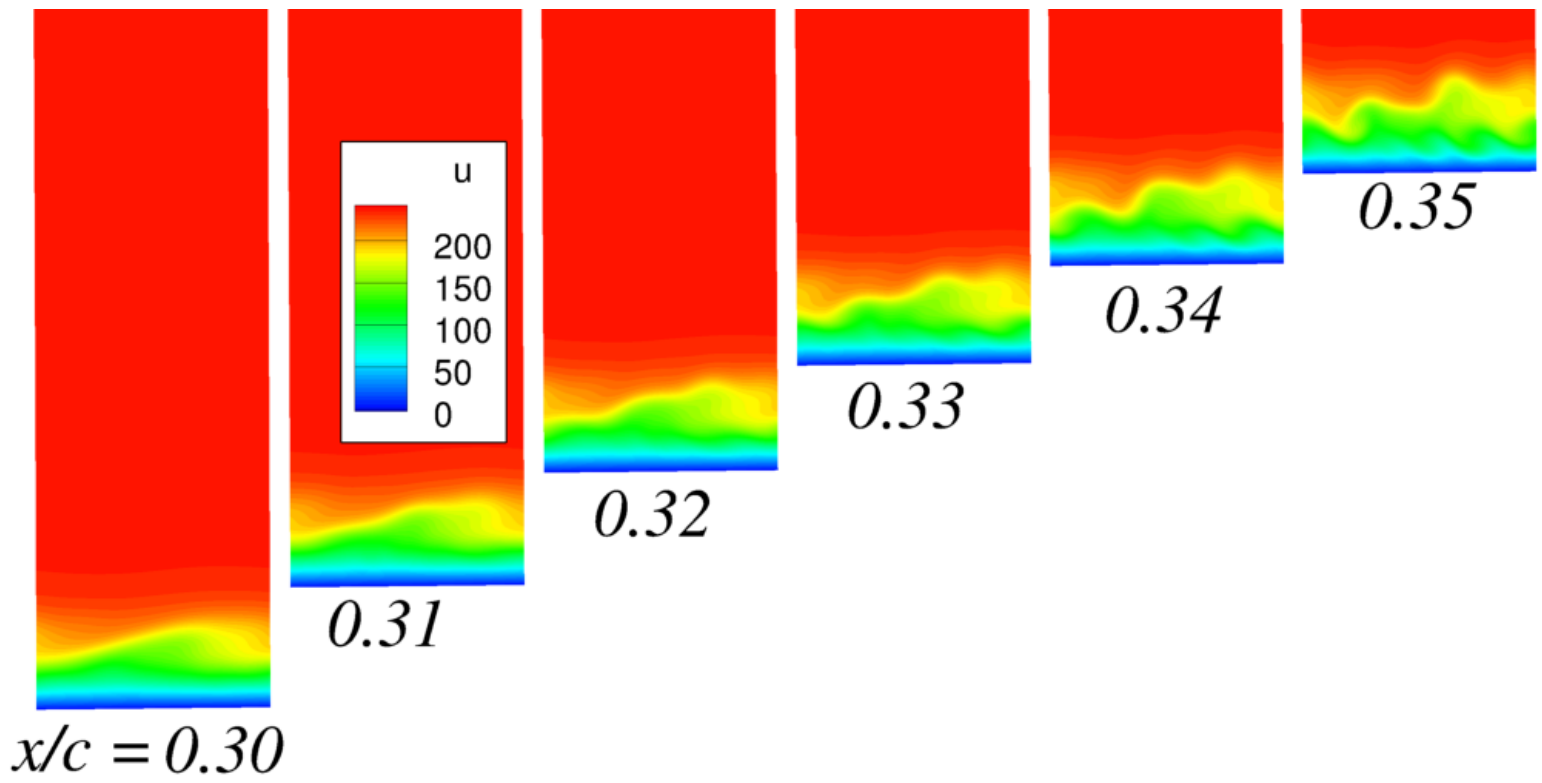

Figure 8. Stationary-frame simulation: Crossplane contours of instantaneous chordwise velocity at select $x$ locations. The range of abscissa in each plot corresponds to one fundamental wavelength of the traveling crossflow mode (i.e., $\lambda=8 \mathrm{~mm}$.)

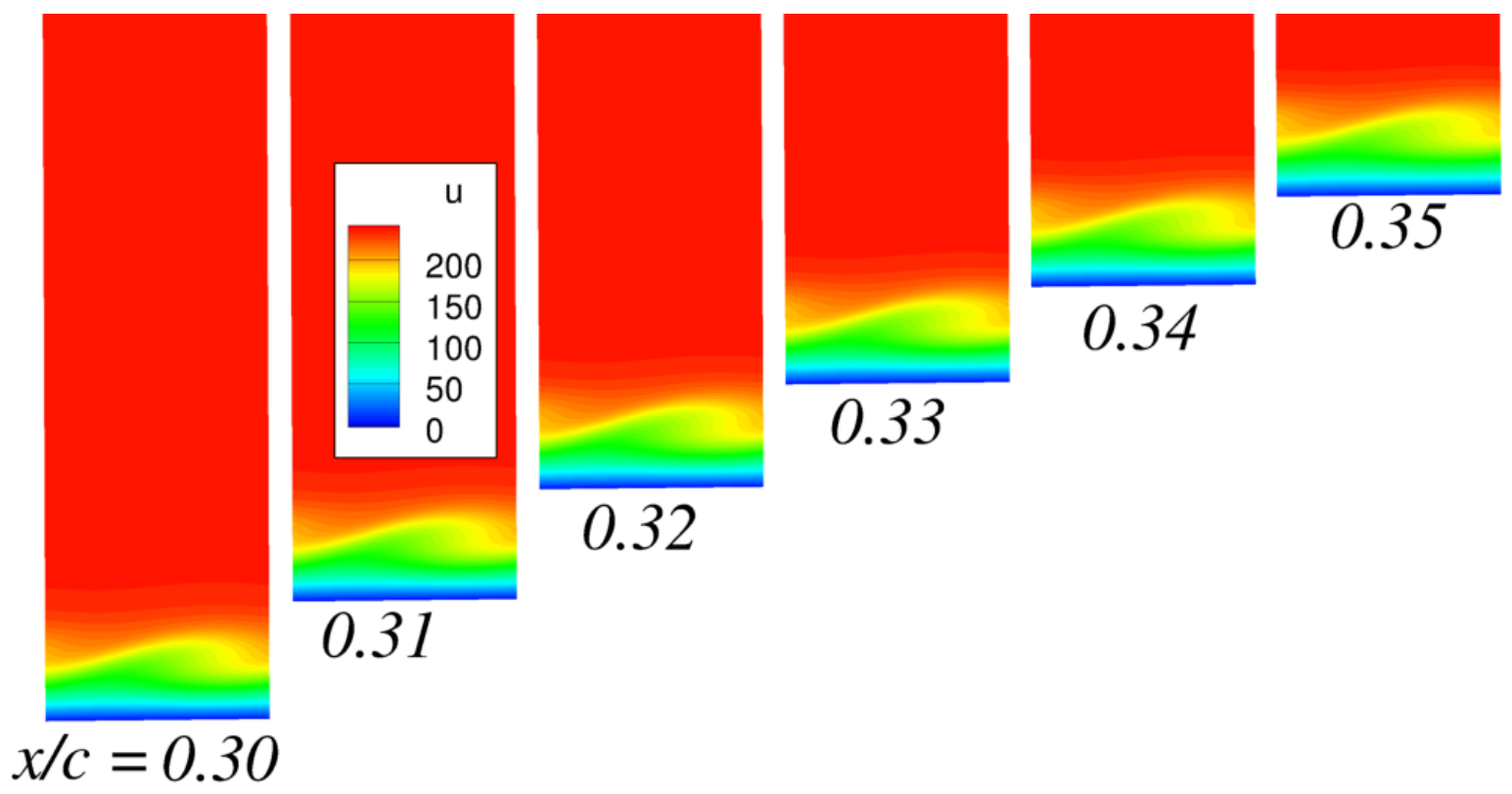

Figure 9. Crossplane contours of instantaneous chordwise velocity at select $x$ locations: primary-only simulation in the stationary frame. 

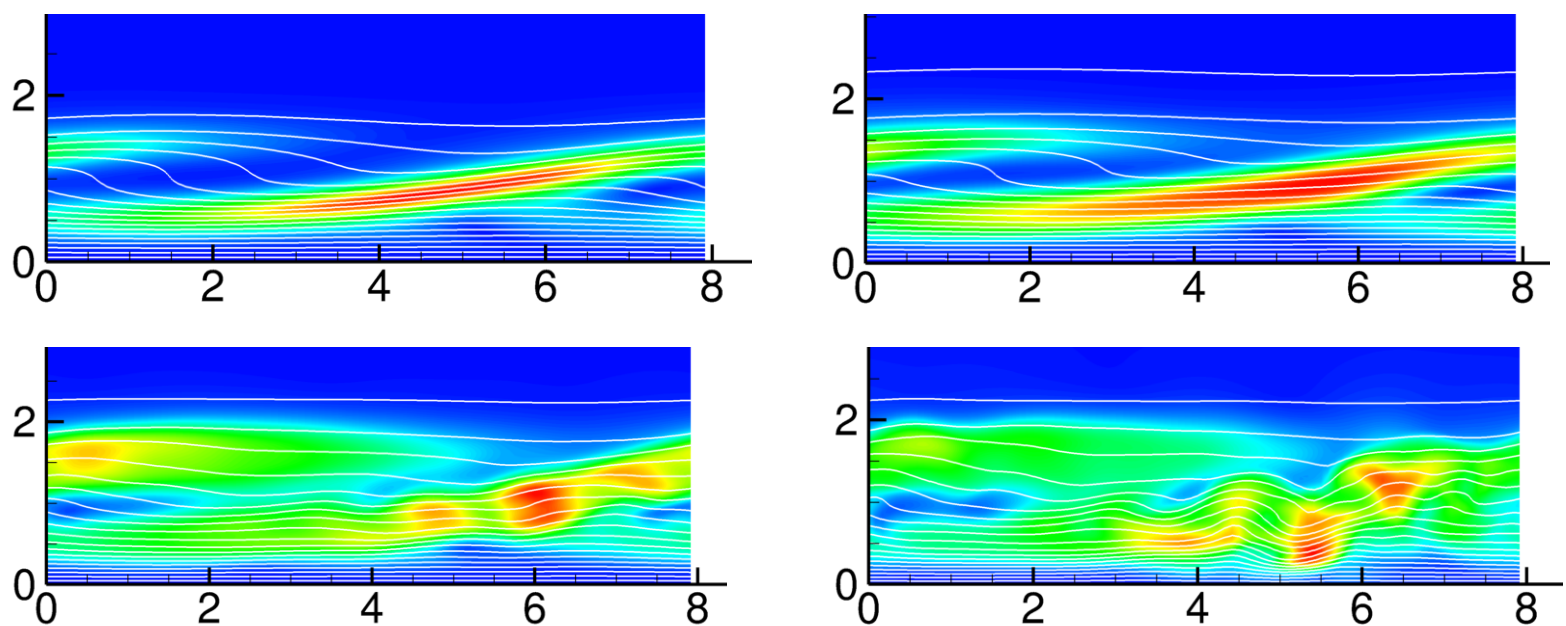

a) Perturbed laminar region ahead of transition onset.

Streamwise locations, $x / c=0.302,0.352,0.385$ and 0.391 from left to right and top to bottom.
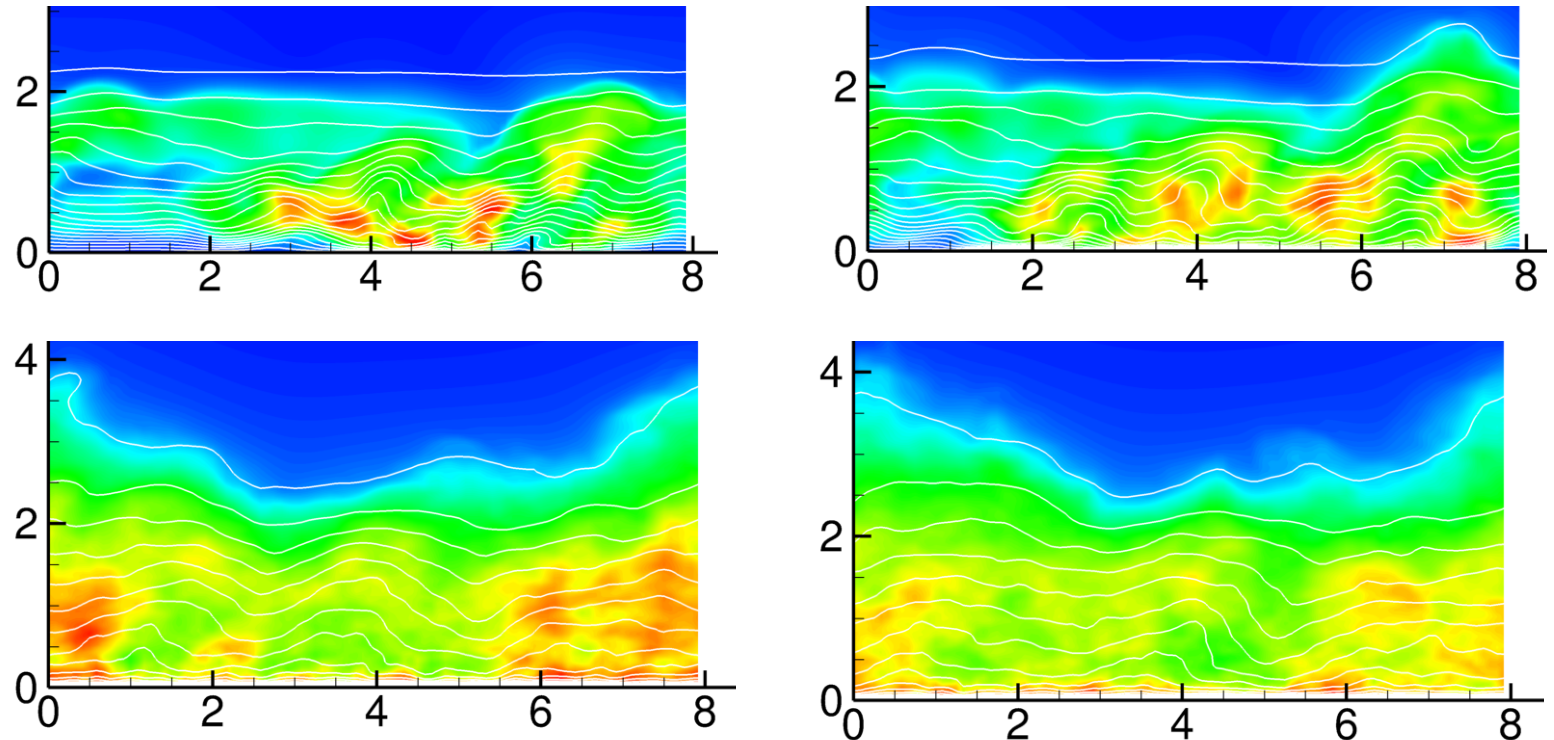

b) Perturbed region during transition

Streamwise locations, $x / c=0.394,0.396,0.403$ and 0.405 from left to right and top to bottom.

Figure 10. Crossplane contours of instantaneous chordwise velocity at select $x$ locations. The range of abscissa in each plot corresponds to one fundamental wavelength of the traveling crossflow made (i.e., $\lambda=8 \mathrm{~mm}$.) Evolution of root-mean-square chordwise velocity perturbation $u$, normalized to have a peak value of unity across the computational domain. (continued). 

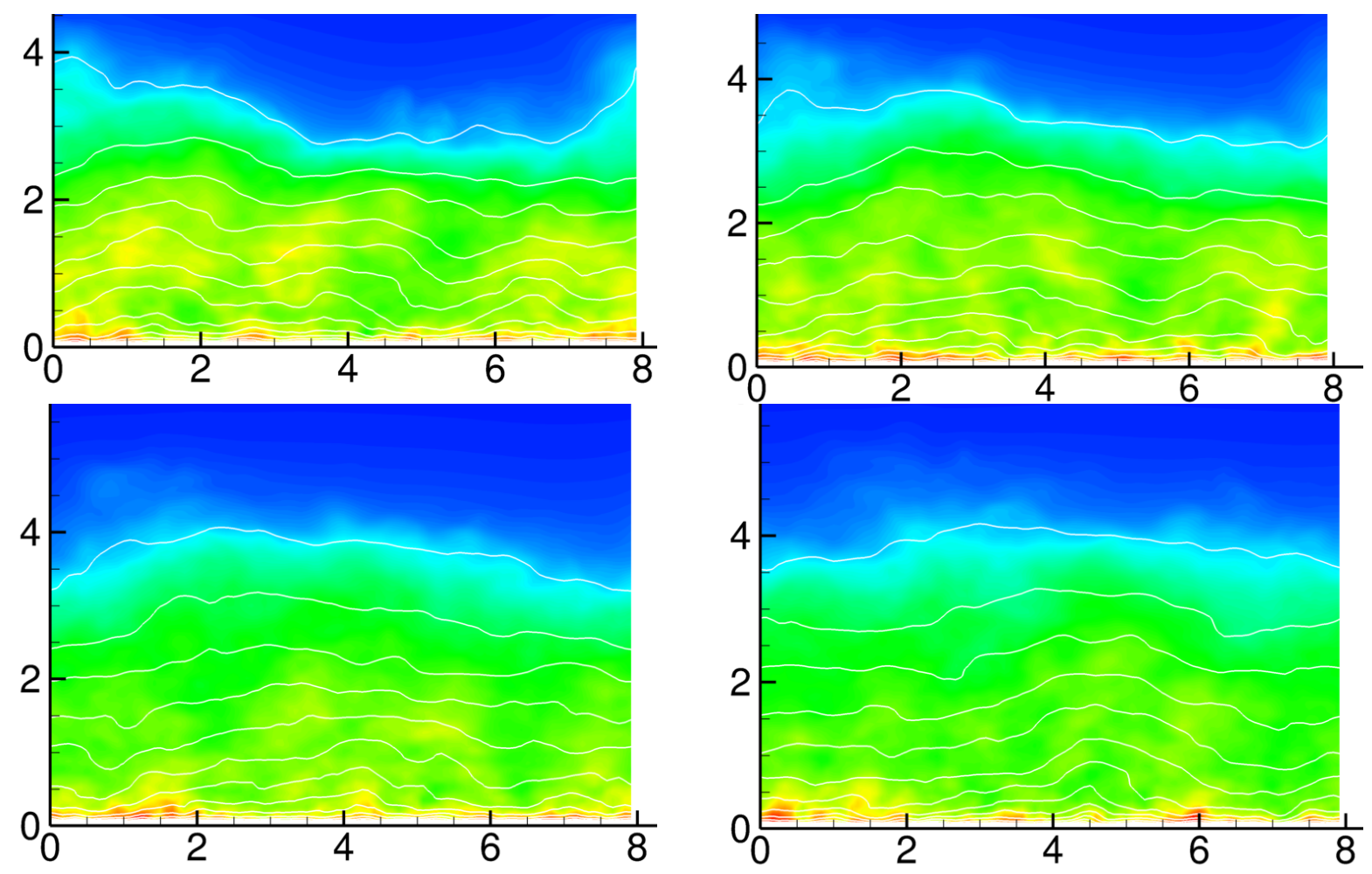

c) Turbulent region

Streamwise locations, $x / c=0.407,0.412,0.416$ and 0.421 from left to right and top to bottom.

Figure 10. Concluded.

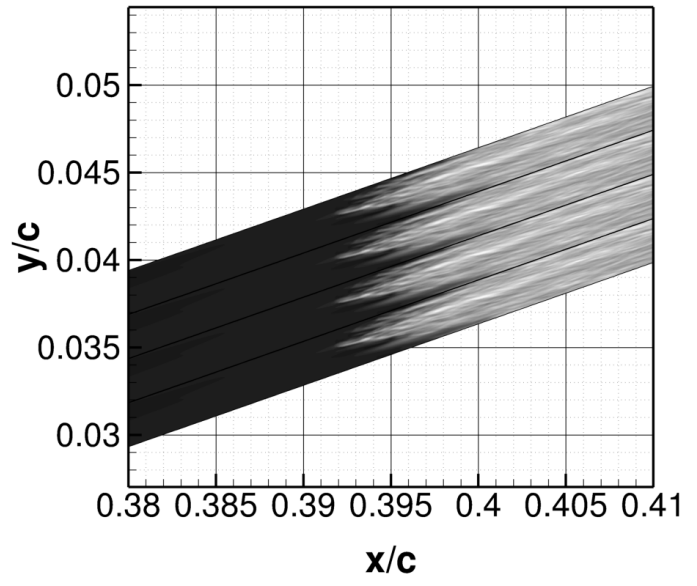

a) Evolution of wall shear in the vicinity of transition onset. Dark shades indicate low shear values corresponding to the laminar boundary layer, whereas light shades on the right indicate substantially higher shear representative of the turbulent state.

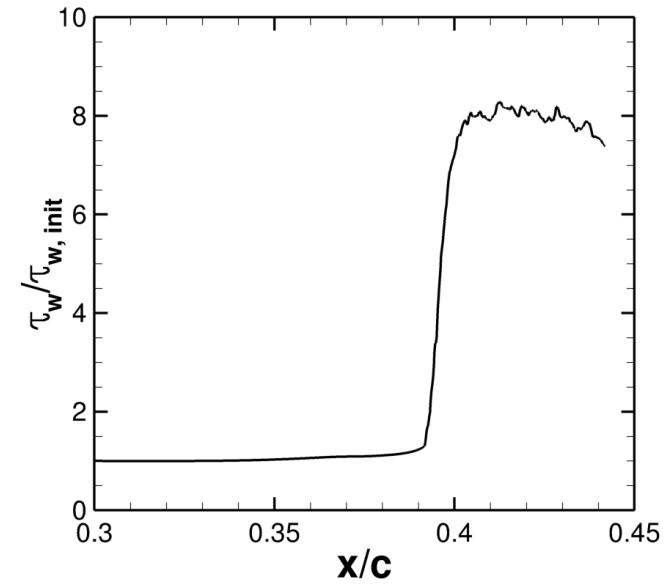

b) Mean wall shear associated with chordwise velocity.

Figure 11. Wall shear variation across transition region. 


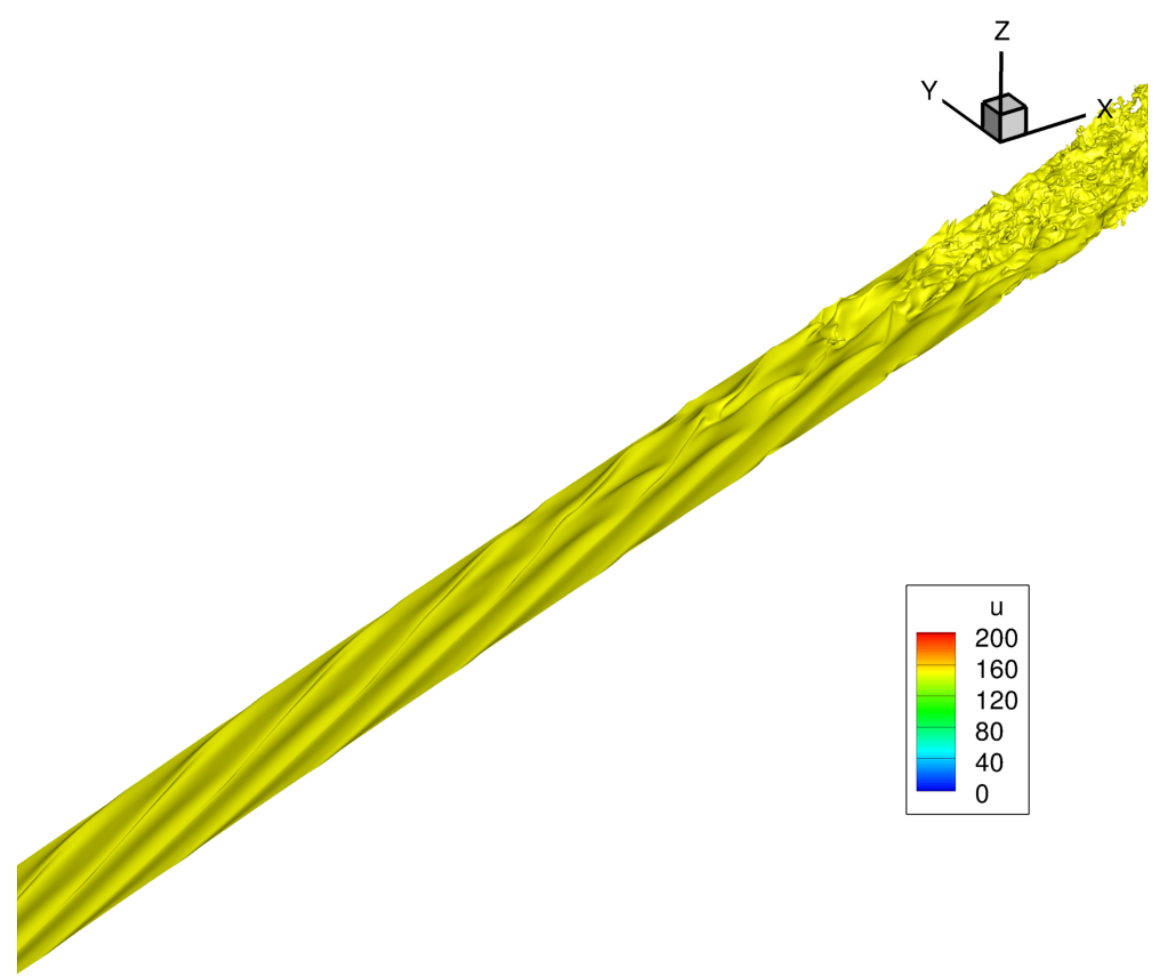

a) $x / c=0.373-0.407$

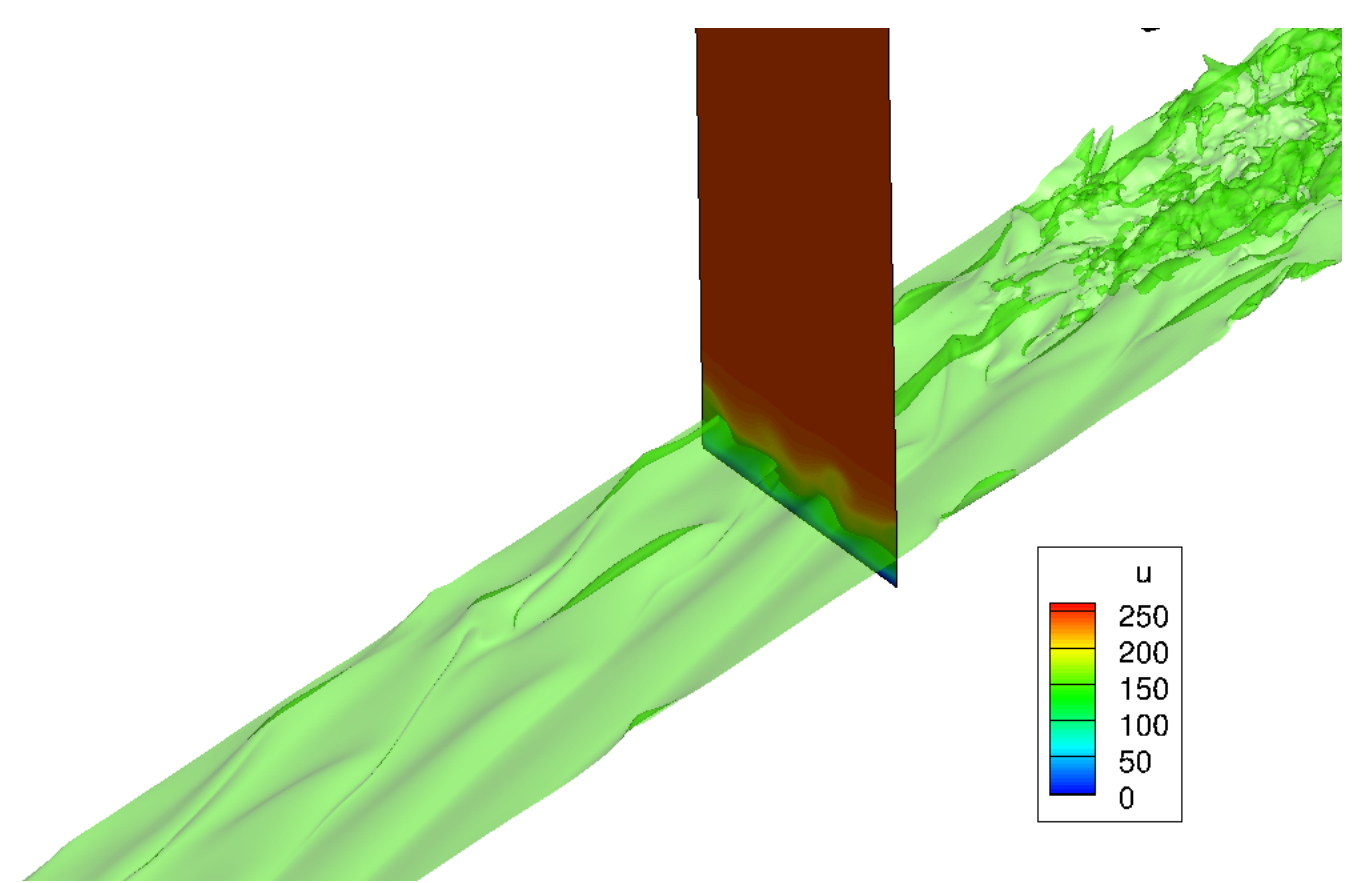

b) Zoomed in view of an aft segment of the region in part (a); the crossplane image corresponds to $x / c=0.394$.

Figure 12. Iso-surface of instantaneous chordwise velocity corresponding to $u / U=0.63$. 